\title{
CONVERGENT DEBONDING OF FILMS AND FIBERS
}

\author{
M. Y. HE' ${ }^{1}$ A. G. EVANS ${ }^{2}$ and J. W. HUTCHINSON ${ }^{2}$ \\ 'Materials Engineering Department, University of California, Santa Barbara, California and 'Division of \\ Engineering and Applied Sciences, Harvard University, Pierce Hall, Cambridge, MA 02138, U.S.A.
}

(Received 16 August 1996)

\begin{abstract}
Debonding of films attached to substrates as well as fibers embedded in matrices typically involves initiation, steady-state propagation and a final transient as the debond converges on an edge or another debond. The emphasis in this paper is on the mechanics of the transient. Under most circumstances, a converging debond crack is characterized by an energy release rate that approaches zero, causing the crack to arrest without attaining full debonding. The relevance of this phenomenon will bo discussed with reference to the measurement of toughness for thin film interfaces and the overall stress-strain behavior of ceramic matrix composites. (C) 1997 Acta Metallurgica Inc.
\end{abstract}

\section{INTRODUCTION}

Residually stressed thin films debond from attached substrates when the stress and/or the film thickness becomes sufficiently large. The qualitative relation between the energy release rate $G$ motivating growth of a plane strain interface crack is sketched in Fig. 1(a) for a film with a uniform residual stress $\sigma$ and thickness $t$. When the crack tip is remote from the edges of the film, steady-state propagation conditions prevail with energy release rate

$$
G_{\mathrm{ss}}=\frac{1-v_{1}^{2}}{2} \frac{\sigma^{2} t}{E_{1}}
$$

where $E_{1}$ and $v_{1}$ are the Young's modulus and Poisson's ratio of the film. When the crack is either short or long such that its tip is within several thicknesses of the edge, it "senses" the edge, and $G$ decreases below the steady-state value of equation (1), approaching zero as the distance between the tip and the edge becomes small. Because equation (1) is the maximum energy release rate, the fail-safe criterion against debonding is: $G_{s s}<L_{i}$, where $I_{i}$ is the interface toughness at the relevant mode mixity.

Tests to measure the interface toughness of thin film-substrate systems often employ steady-state conditions $[1,2]$ because the mechanics is simple and because accurate measurement of the crack length is not required. For such tests to be effective, a sharp pre-crack must be introduced. Otherwise, an initiation barrier exists. Often, there is a barrier [Fig. 1(a)] such that the combination of stress and thickness needed to initiate interface crack propagation exceeds that required for steady-state growth, causing $G_{\mathrm{ss}}>\Gamma_{\mathrm{i}}$. The debond event is then dynamic, with the crack arresting when the tip approaches the far edge. Such a test would be designated unsuccessful in the sense that $G_{\mathrm{ss}}$ [equation (1)] cannot be identified with $\Gamma_{i}$. However, when the relation between $G$ and the crack length as the tip approaches the far edge is known, quantitative conclusions about $\Gamma_{\mathrm{i}}$ can be made from measurements of the distance between the edge and the arrested crack tip. One purpose of the present paper is to supply the nccessary solutions.

The crack arrest phenomenon is depicted in Fig. 2 [3]. It comprises a Ni-polymer bilayer on a polymer monolayer well bonded to a stiff substrate. The interface of interest is that joining the two polymers. The residual tension in the top polymer film is not sufficient to debond the interface. Therefore, a superlayer of $\mathrm{Ni}$ with strong adherence to the polymer and a large residual tension has been deposited. It is this stress which supplies the energy needed to debond the polymer-polymer interface. This occurs when the Ni film becomes sufficiently thick that super critical conditions are attained. Further details are given in Section 3.3. Here, it is noted that removal of an initiation barrier requires a complex additional step in specimen preparation [2]. Consequently, determination of the interface toughness from the arrest location of the debond cracks facilitated the testing and also gave more robust data.

Related transient interface debonding is displayed by fibers embedded in brittle matrices. The behavior illustrated in Fig. 1(b) applies to a composite layer with uni-directional fibers carrying an overall stress $\bar{\sigma}$ parallel to the fibers. When matrix cracks develop, a debond propagates up the fiber. The combined effects of applied stress, friction and residual stresses must be superimposed for complete analysis of debond evolution $[4,5]$. Here, only the contribution due to the applied stress $\bar{\sigma}$ will be addressed to highlight the convergence effect. There is again an initiation barrier because the energy release rate for very short debonds emerging from the tip of the matrix crack is below its 
(a)

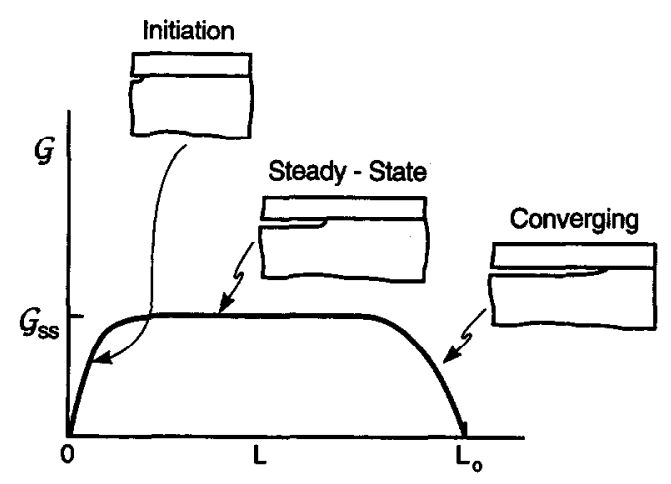

Film/Substrate Debonding (b)

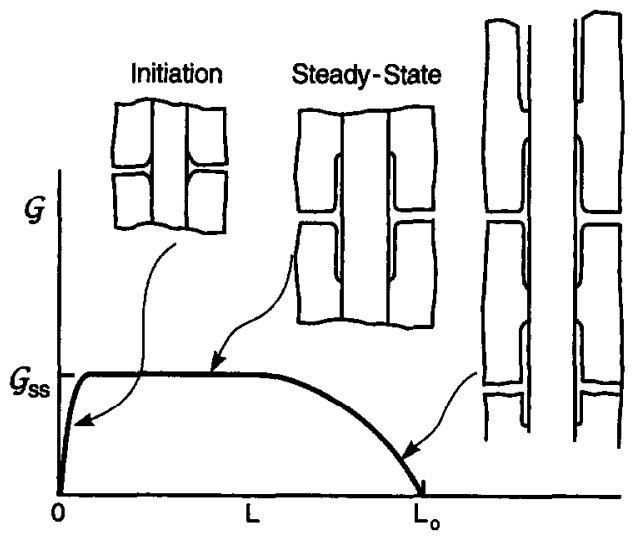

Fiber/Matrix Debonding

Fig. 1. Three stages of debonding for films and fibers.

steady-state magnitude [5]. In steady-state, the energy release is given by [4]:

$$
G_{\mathrm{ss}}=\frac{(1-f)}{4 f^{2}} \frac{\left(1-v^{2}\right) \bar{\sigma}^{2} R}{E},
$$

where $f$ is the volume fraction of the fibers and $R$ is their radius. This particular expression is restricted to fibers and matrix having the same isotropic elastic constants, $E$ and $\nu$. The effects of elastic mismatch are addressed later. As a debond spreads along the fiber and converges on another debond extending in the opposite direction from the neighboring matrix crack, the energy release rate approaches zero, as indicated in Fig. 1(b). The converging debonds arrest leaving a portion of the fiber still attached to the matrix. This behavior has implications for the overall stress-strain behavior of the composite discussed in Section 4.

\section{THE ASYMPTOTIC LIMIT FOR A CONVERGING DEBOND}

Consider two converging crack tips sufficiently close that the only relevant length is the distance $\Delta L$ separating them, as depicted by the plane strain problem in Fig. 3. This limit also provides a reasonable approximation for a debond tip approaching a free edge, discussed in connection with

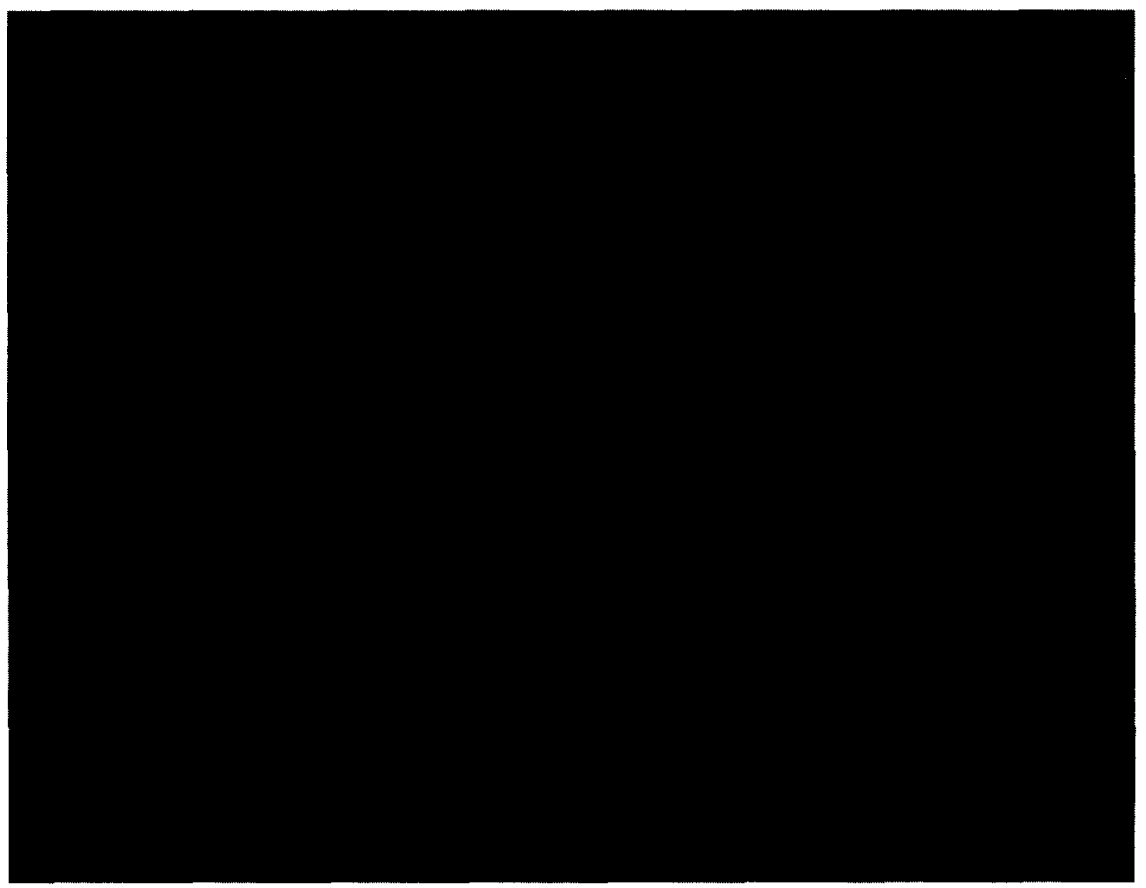

Fig. 2. Thin polymer lines on a Si substrate with a SAM interface, plus a Ni superlayer. The intact ligament length gives the interface toughness. 


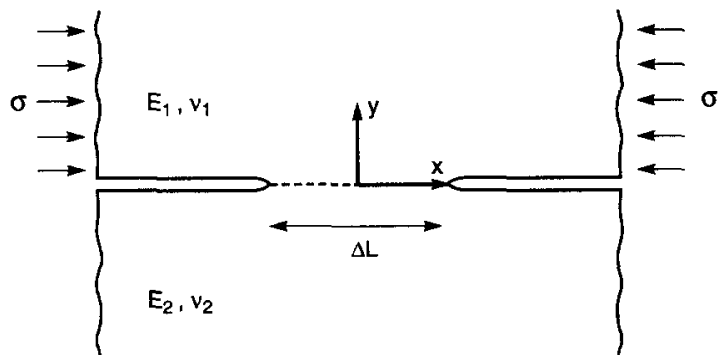

Fig. 3. Geometry and loading for asymptotic debonding problem.

Fig. 1(a). Moreover, it applies to debonds converging along a fiber, because plane strain is attained when the debond tips are close. Subsequent numerical results for the energy release rate will be seen to approach this asymptotic limit in the transition between steady-state and full debonding.

The solution to the problem of isotropic elastic half-planes joined along the interface on $y=0$, $|x|<\Delta L / 2$ and loaded by a remote stress $\sigma_{11}=-\sigma$ in the upper half-plane can be obtained using the complex variable method of elasticity. Similar solutions have been presented by Rice and Sih [6] and Erdogan [7]. The two Dundurs parameters, $\alpha$ and $\beta$, measuring the elastic mismatch between the two half-planes enter the solution, as does the so-called oscillation index $\epsilon$ defined in terms of $\beta$ by

$$
\epsilon=\frac{1}{2 \pi} \ln \left(\frac{1-\beta}{1+\beta}\right)
$$

In plane strain, the Dundurs parameters are given by

$$
\begin{aligned}
& \alpha=\frac{\bar{E}_{1}-\bar{E}_{2}}{\bar{E}_{1}+\bar{E}_{2}} \\
& \quad \text { and } \beta=\frac{1}{2} \frac{\mu_{1}\left(1-2 v_{2}\right)-\mu_{2}\left(1-2 v_{1}\right)}{\mu_{1}\left(1-v_{2}\right)+\mu_{2}\left(1-v_{1}\right)},
\end{aligned}
$$

where $\bar{E}=E /\left(1-v^{2}\right)$ and $\mu=E /(2(1+v))$ is the shear modulus.

The tractions acting on the bonded portion of the interface are given by

$$
\sigma_{22}+\mathrm{i} \sigma_{12}=-\frac{\mathrm{i}(1-\alpha) \sigma}{2 \sqrt{1-\beta^{2}}} \frac{x \mathrm{e}^{-\mathrm{i} \epsilon \ln \left(\frac{\Delta L-2 x}{\Delta L+2 x}\right)}}{\sqrt{\Delta L^{2}-4 x^{2}}} .
$$

For the crack with tip at $x=-\Delta L / 2$, stress intensity factors $K_{1}$ and $K_{2}$ are defined such that on the interface a short distance $r$ ahead of the tip [8]

$$
\sigma_{22}+\mathrm{i} \sigma_{12}=\frac{\left(K_{1}+\mathrm{i} K_{2}\right)}{\sqrt{2 \pi r}} r^{\mathrm{ic}} \text {. }
$$

For the converging debond crack advancing from the left in Fig. 3, the stress intensity factors are

$$
K_{1}+\mathrm{i} K_{2}=\frac{\mathrm{i}(1-\alpha) \sigma \sqrt{\pi \Delta L}(\Delta L)^{-\mathrm{i}}}{4 \sqrt{2\left(1-\beta^{2}\right)}} .
$$

The associated energy release rate is given by

$$
G=\frac{1-\beta^{2}}{E_{*}}\left(K_{1}^{2}+K_{2}^{2}\right)=\frac{(1-\alpha)^{2} \sigma^{2} \pi \Delta L}{32 E_{*}}
$$

where $E_{*}^{-1}=(1 / 2)\left(\vec{E}_{1}^{-1}+\bar{E}_{2}^{-1}\right)$. This result for $G$ normalized by $G_{\mathrm{ss}}$ in equation (1) gives

$$
\frac{G}{G_{\mathrm{ss}}}=\frac{\pi}{16}(1-\alpha) \frac{\Delta L}{t} .
$$

Note that $G$ vanishes as the debond tips converge. The energy release rate is a strong function of the elastic mismatch $\alpha$, but it is independent of $\beta$. The mode measure $\psi$, defined as $\tan \psi=\sigma_{12} / \sigma_{22}$ a distance $r$ ahead of the tip, is obtained from equation (7) as

$$
\psi=\frac{\pi}{2}+\epsilon \ln \left(\frac{r}{\Delta L}\right)
$$

When $\beta=0$, the crack tip loading is mode $I$, with $K_{2}=(1-\alpha) \sigma \sqrt{\pi \Delta L} /(4 \sqrt{2})$. Otherwise, the tip experiences mixed-mode conditions.

\section{THIN FILM DEBONDING}

\subsection{Homogeneous films under uniform residual stress}

Various thin film debonding scenarios can be imagined. Figures 4(a)-(c) illustrate the three considered here. The simplest to represent is that depicted in Fig. 4(a) where the elastic energy release in a film with a uniform tensile pre-stress $\sigma$ drives two interface cracks converging upon each other from opposite directions. The interface stress intensity

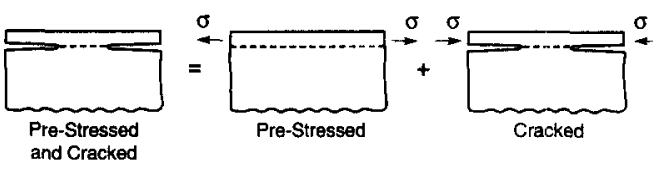

(a) Symmetric Debonds
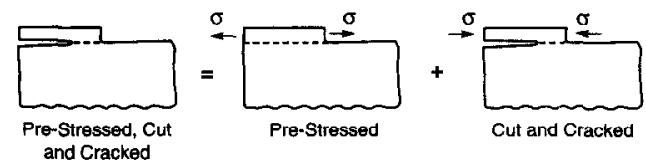

(b) Debond Approaching Film Edge
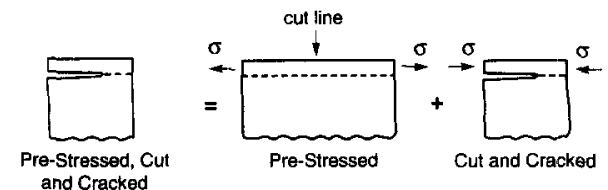

(c) Debond Approaching Film/Substrate Edge

Fig. 4. The scenarios for convergent debonding of thin films. 
factors and the energy release rates are determined from the solution to the second of the two elasticity problems indicated on the right in Fig. 4(a). This problcm is symmetric with respect to the $y$-axis. Primary attention, however, is directed towards the second scenario, Fig. 4(b), whereby a uniformly pre-stressed film is terminated at $x=0$, exposing a free edge. An interface crack propagates from the left towards the free edge. Again, the second elasticity problem on the right in Fig. 4(b) needs to be solved as the crack converges upon the free edge. In this case, the compressive stress $\sigma$ acting on the right hand free edge of the film is an essential feature of the solution. Since only elastic behavior is considered, the sequence of cutting followed by interface cracking need not be treated as separate events. In the third scenario, Fig. 4(c), both the pre-stressed film and the substrate is terminated along $x=0$, with the debond crack approaching the free edge. Results for this scenario have not been computed. They are expected to be similar to Fig. 4(b), especially when the substrate is stiff compared with the film.

Results have been computed using a finite element model of the problems depicted in Fig. 4. The width of the model is taken to be $300 t$, while the depth of the substrate is taken as $20 t$. The bottom of the substrate is constrained against vertical displacement but free to displace horizontally. A highly refined mesh is used in the vicinity of the crack tip. The $J$-integral is used to evaluate $G$, and $\psi$ is computed by fitting theoretical crack tip opening and shear displacement amplitudes to the numerical values. An excellent check on the accuracy of the model is provided by the analytical results for the steady-state limit [equation (1)] which is approached at sufficiently large $\Delta L / t$. The corresponding limit for the measure of mode mixity is $\psi=\omega(\alpha)$, where $\omega$ is tabulated by Suo and Hutchinson [9]; in the absence of elastic mismatch, $\omega(0)=52.1^{\circ}$. Trends in $G$ for four mismatch parameters $\alpha$ are presented in Fig. 5(a) for the second scenario described above. Apart from the curve for $\alpha=-1$ corresponding to the limit of a rigid substrate, the results have been computed with $\beta=0$. The companion results for the mode-mixity measure, $\psi$, for the non-rigid substrates are displayed in Fig. 5(b).

The debond begins to sense the edge of the film when the tip is within 5-20 times the film thickness, depending on the elastic mismatch. This distance is surprisingly large. It is this feature of the converging debond which allows graceful arrest and makes measurement of interface toughness feasible. Figure 6(a) illustrates the approach to the asymptotic limit [equation (9)] for both symmetrically converging debonds and a debond converging on an edge for a mismatch where the film is compliant relative to the substrate, $\alpha=-1 / 2$. For this mismatch, the asymptotic result becomes accurate for the symmetrically convergent debonds when $\Delta L / t$ is about $1 / 2$, but it somewhat overestimates the energy release rate for a (a)

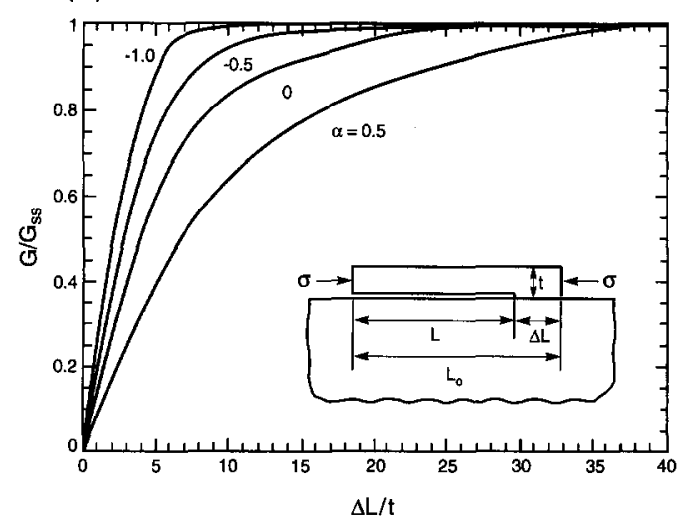

(b)

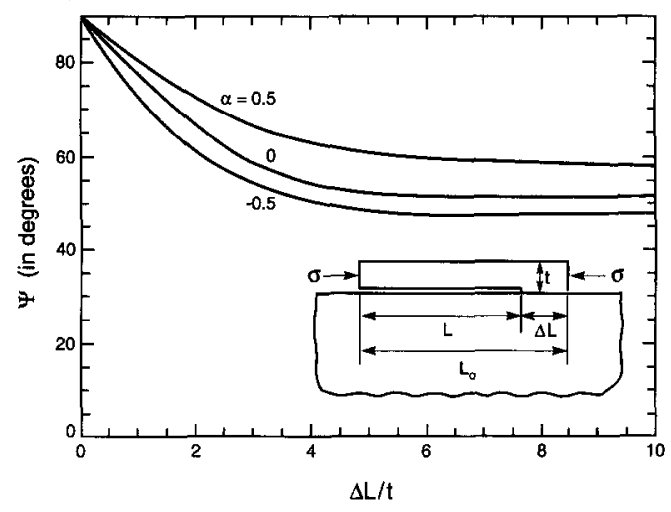

Fig. 5. Energy release rate (a) and measure of mode mixity (b) for convergent debonds.

debond converging on a free edge. A comparison with the asymptotic result over the full mismatch range is given in Fig. $6(\mathrm{~b})$ at $\Delta L / t=2$, for which there is essentially no difference between the numerical results for the two cases. Another important implication of Fig. 6 is that, when the remaining ligament is greater than about $t / 2$, the energy release rate of a symmetrically converging debond is essentially indistinguishable from that for a debond converging on a free edge the same distance away. In other words, the debond is unable to distinguish another debond from a free edge until it has approached to within a film thickness.

Converging debond phenomena have been observed for films delaminating under nominally axisymmetric conditions with an external interface crack propagating inward debonding the remaining circular ligament [10]. As in the plane strain cases analysed here, the energy release rate approaches zero as the remaining ligament is debonded [11]. Gao's [12] result for the asymptotic limit when the ligament radius $a$ becomes small compared to the film thickness is $G=(1-v) \sigma^{2} a$ l $[\pi(1+v) E]$ for the case in which the film and substrate have no elastic mismatch and $\sigma$ is the equi-biaxial pre-stress. 
3.2. Homogeneous films under non-uniform residual stress

For completeness, results are derived which enable the determination of $G$ and $\psi$ for the case [Fig. 4(b)] in which a crack converges upon a cut edge when the film has a residual stress that varies through its thickness, $\sigma(y)$. Let $\bar{\sigma}=1 / t \int_{0}^{t} \sigma(y) \mathrm{d} y$ be the average residual stress and $M$ be the moment of distribution about the film midplane, i.e. $M=\int_{0} \sigma(y)(y-t / 2) \mathrm{d} y$. The results presented in the previous sub-section apply for the contribution due to $\bar{\sigma}$. Results for $G$ and $\psi$ for $M$ with $\bar{\sigma}=0$ are given in Fig. 7 for several $\alpha$ with $\beta=0$. Note that a two-part composition of the solution [such as that in Fig. 4(b)] applies here also, but with $M$ acting in the opposite sense (Fig. 7). The results apply when $M>0$, such that the residual stress in the film is tensile at its top surface and compressive at the substrate interface. The debond crack is open; it would be closed when $M<0$. In steady-state,

$$
\bar{G}_{\mathrm{ss}}=\frac{1 \quad v_{1}^{2}}{E_{1}} \frac{6 M^{2}}{t^{3}}
$$

(a)

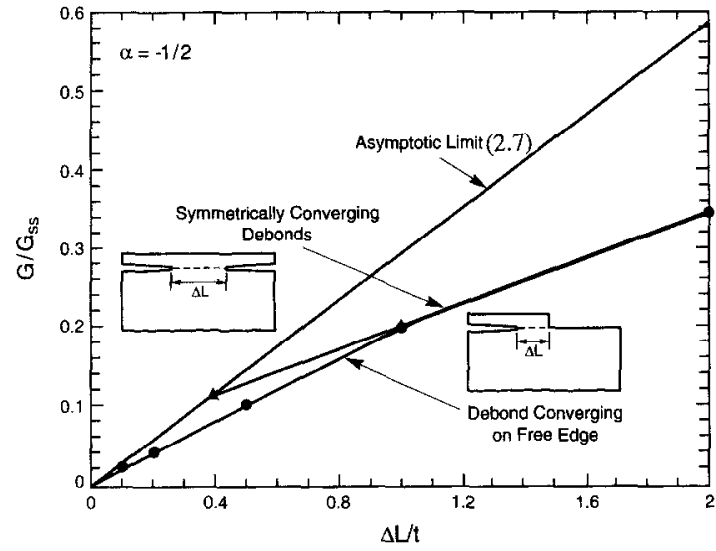

(b)

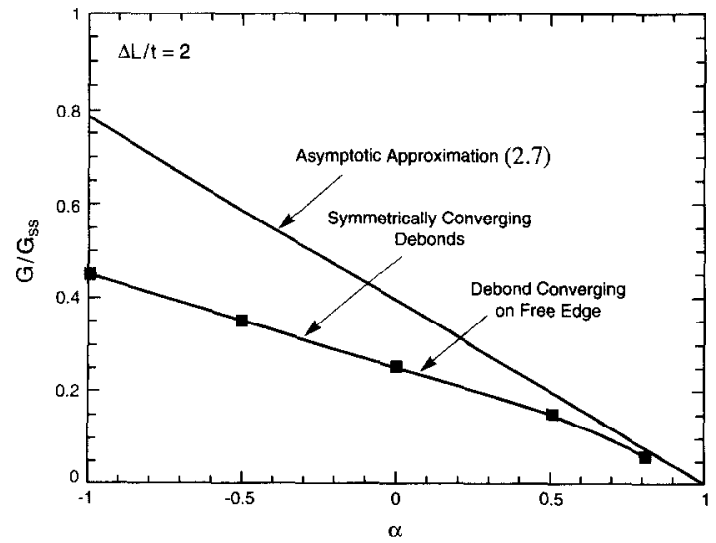

Fig. 6. Comparison of numerical results and asymptotic formula (9) for two cases of convergent debonds: (a) $\alpha=-1 / 2$; (b) $\Delta L / t=2$. (a)

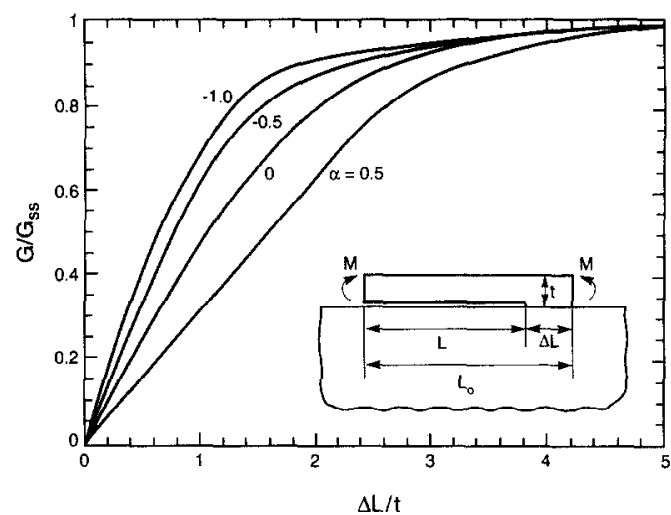

(b)

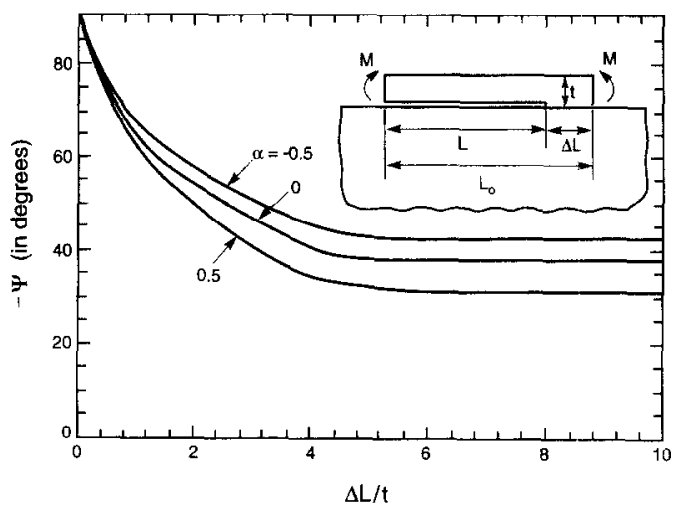

Fig. 7. Energy release rate (a) and measure of mode mixity (b) for converging debond for residual stress equivalent to a moment per unit length $M$.

while $\quad \psi_{\mathrm{ss}}=-\pi / 2+\omega(\alpha)$ when $M>0$, but $\psi_{\mathrm{ss}}=-\pi / 2$ (mode II) when $M<0$. The most notable feature brought out by Fig. 7 , relative to the results for $\bar{\sigma}$ in Fig. 5, is the considerably smaller domain in which the interface crack departs from steady state.

Under combined $\bar{\sigma}$ and $M, G_{\mathrm{ss}}$ and $\psi_{\mathrm{ss}}$ are given by

$$
G M a_{s \mathrm{~s}}=\frac{1-v_{1}^{2}}{E_{1}}\left[\frac{\bar{\sigma}^{2} t}{2}+\frac{6 M^{2}}{t^{3}}\right]
$$

and

$$
\tan \psi_{\mathrm{ss}}=\frac{\sqrt{12} M \cos \omega+\bar{\sigma} h^{2} \sin \omega}{-\sqrt{12} M \sin \omega+\bar{\sigma} h^{2} \cos \omega}
$$

When the crack is not in steady state, $G$ cannot be obtained by an addition of the respective energy release rates (due to $\bar{\sigma}$ and $M$ ). Then, $G$ and $\psi$ must be obtained from a linear superposition of the stress intensity factors. When $\beta=0$, the respective contributions are obtained from Figs 5 or 7 using $K_{1}=\sqrt{E * G} \cos \psi$ and $K_{2}=\sqrt{E * G} \sin \psi$. Then, the desired results are generated using $G=\left(K_{1}^{2}+K_{2}^{2}\right) / E_{*}$ and $\tan \psi=K_{2} / K_{1}$. 


\subsection{Two-layer films: A stressed metal layer on top of a compliant polymer layer}

It becomes increasingly difficult to present comprehensive results for multi-layer delamination. Closed form results under steady-state conditions can be obtained for $G$, but not for $\psi$. General results for converging debonds where the film is multi-layered are even harder to obtain, but a surprisingly accurate and simple formula for the energy release rate can be derived for a two-layer film comprised of a stiff pre-stressed layer attached to a compliant underlayer. We begin by presenting a limited set of finite element results for a two-layer film having individual layers representative of the epoxy- $\mathrm{Ni}$ system discussed in the Introduction, illustrated in Fig. 2 [3]. The purpose of these experiments was to study debonding of polymer-polymer interfaces with systematically varying interface chemistry. For this purpose, a self assembled monolayer (SAM) with either a $\mathrm{CH}_{3}$ or a $\mathrm{COOH}$ end member, has been placed on a $\mathrm{Si}$ substrate. An epoxy layer of thickness $t_{1}=1 \mu \mathrm{m}$ has been superposed. The top $\mathrm{Ni}$ layer, deposited by vapor deposition, is subject to an intrinsic residual tension of the order of $1 \mathrm{GPa}$. It is the elastic energy stored in this layer that drives the debond crack, when the layer thickness exceeds a critical value. The mechanics model analyzed numerically for this case is shown in the insert in Fig. 8. For the purpose of calculating $G$, the SAM is ignored because it is very thin. The interface lies between the epoxy layer $\left(E_{1}\right.$, $\left.v_{1}, t_{1}\right)$ and the infinitely thick $\mathrm{Si}$ substrate $\left(E_{2}, v_{2}\right)$. The Ni layer $\left(E_{3}, v_{3}, t_{3}\right)$ on top has a residual tension, $\sigma$. The epoxy layer also supports a small residual tension, but the elastic energy stored in this layer is less than $1 \%$ of that in the Ni layer and can be ignored.

The numerical results in Fig. 8 display the energy release rate $G$ as a function of $\Delta L / t_{1} \equiv\left(L_{0}-L\right) / t_{1}$ for two values of $E_{1} / E_{3}$ representative of the expected moduli ratio of an epoxy to $\mathrm{Ni}$. The ratio $E_{3} / E_{2} \mathrm{Ni}$ to silicon is about unity, the Poisson ratios have been

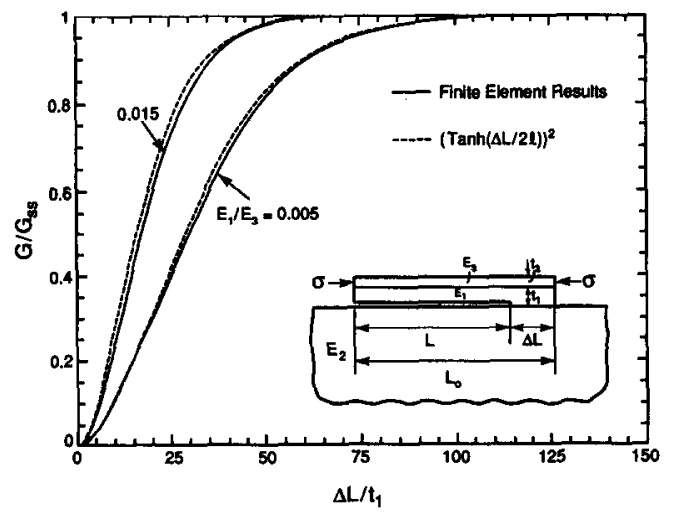

Fig. 8. Energy release rate for convergent debonding for a bi-layer film where the top layer is subject to a residual stress $\sigma$, with $t_{3} / t_{1}=0.4$ and the other parameters are specified in the text.

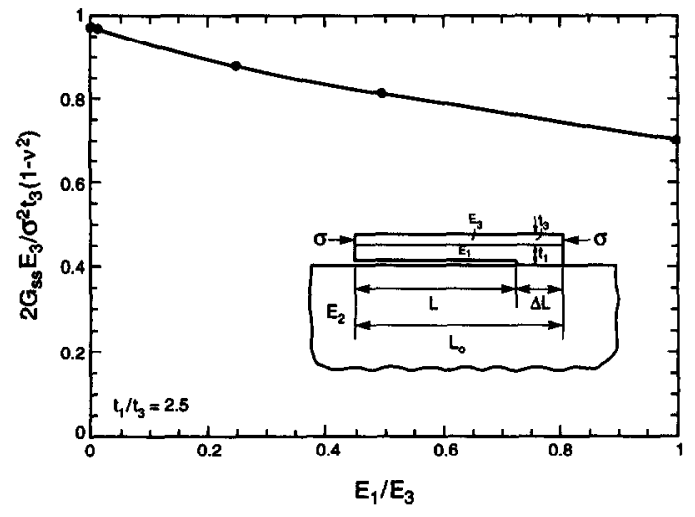

Fig. 9. Effect of modulus ratio $E_{1} / E_{3}$ on steady-state energy release rate of a bi-layer film with $t_{3} / t_{1}=0.4$ and only the top layer subject to residual tension.

chosen as $v_{1} \cong 0.5, v_{2}=0.25$ and $v_{3}=0.33$, and the ratio of the two-layer thicknesses is taken to be $t_{3} / t_{1}=0.4$, corresponding to the films in Fig. 2 . In Fig. $8, G$ has been normalized by $\left(1-v_{3}^{2}\right) \sigma^{2} t_{3} /\left(2 E_{3}\right)$ which is slightly greater than the steady-state energy release rate $G_{\text {ss }}$, as can be seen in Fig. 9. Because the epoxy layer is so compliant compared with the $\mathrm{Ni}$ layer, almost all of the residual elastic energy stored in the $\mathrm{Ni}$ layer is released by the debonded two-layer film (subject to plane strain constraint in the out-of-plane direction). As the stiffness of layer \#1 increases compared to that of layer \#3, more elastic energy remains in the debonded two-layer film. Nevertheless, even when $E_{3}=E_{1}$, Fig. 9 shows that $G_{\text {ss }}$ is only reduced by $25 \%$ below that estimated upon assuming that all the elastic energy in the top layer is released.

The result derived in the Appendix for the two-layer system shown in the insert in Fig. 8 is

$$
\frac{G}{G_{\mathrm{ss}}}=\tanh ^{2}\left(\frac{\Delta L}{2 l}\right) \text { where } l=\sqrt{\frac{\bar{E}_{3} l_{1} l_{3}}{\mu_{1}}}
$$

with $\quad \bar{E}_{3}=E_{3} /\left(1-v_{3}^{2}\right)$ and $\mu_{1}=E_{1} /\left[2\left(1+v_{1}\right)\right]$. Equation (14) is expected to be accurate if the shear stiffness of layer 1 is small compared to the extensional stiffness of layer 3 such that $l$ is large compared to the total thickness of the film. Predictions obtained from equation (14) for the two cases considered in Fig. 8 are included there as dashed line curves. The simple formula clearly provides a reasonably accurate description of the energy release rate of the converging debond.

The curves in Fig. 8 for the debond converging on the free edge of the film show that $G$ departs from the steady-state limit at large $\Delta L / t_{1}$, especially when the lower of the two layers is relatively compliant. For example, for $E_{1} / E_{3}=0.005$, the interface crack first senses the free edge of the film when its tip is about $75 t_{1}$ from the edge. The experiments on the epoxy- $\mathrm{Ni}$ films, such as those in Fig. 2, show that such distances are characteristic of the ligament size. It is evident 
from the limited set of results presented here that widely disparate moduli of the layers has a major effect on the behavior as the debond converges on the free edge. Another issue yet to be addressed is the role of plastic deformation in the polymer generated by the debond crack tip. The relevance of elastic analysis must be closely examined if the plastic zone becomes comparable in size to the layer thickness.

\section{FIBER DEBONDING}

The trend of energy release rate of a mode II debond spreading along a fiber and approaching a debond progressing in the opposite direction is shown in Fig. 1(b). The focus is on the contribution due to the applied stress $\bar{\sigma}$. A transverse section axisymmetric cell model (Fig. 10) has a fiber radius $R$, and cell outer radius $R_{0}$, such that the volume fraction of the fibers is $f=\left(R / R_{0}\right)^{2}$. The axial load carried by the composite is $\pi R_{0}^{2} \bar{\sigma}$. Matrix cracks have tormed and are spaced $2 L_{0}$. Axisymmetric debond cracks spread along the fiber from the matrix cracks with current length $L$. The debonds are considered to be closed mode II cracks, consistent with the residual stresses present in the composite systems of interest. The elastic properties are taken to be isotropic with $E_{f}, v_{f}$, $E_{\mathrm{m}}$ and $v_{\mathrm{m}}$ as the elastic constants for the fiber and matrix, respectively. The boundary conditions on the lateral sides of the cell mimic the interaction between cellular units in a composite. The shear traction is zero and the radial displacement is constrained to be independent of the axial coordinate such that the average radial component of traction is zero. This boundary condition has been labeled Type II [4].

When the debond length $L$ is larger than about one fiber radius and when $\Delta L \equiv L_{0}-L$ is sufficiently large compared to $R$, the interface crack propagates in steady-state ( $G$ independent of $L$ ). (In this section the distance between the debond crack tips is taken

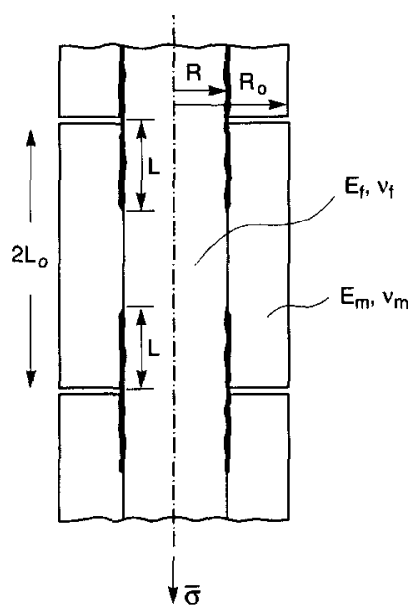

Fig. 10. Cylindrical cell model of a uni-directional ceramic matrix composite with fiber-matrix debonds emerging from neighboring matrix cracks and converging towards each other.

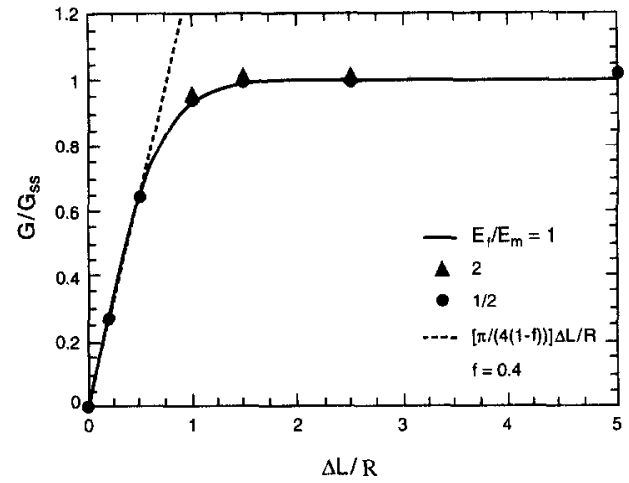

Fig. 11. Energy release rate for debond crack tips approaching each other along a fiber and comparison with the asymptotic result (18).

to be $2 \Delta L$ rather than $\Delta L$, as in Sections 2 and 3.) When there is no elastic mismatch between the fiber and matrix, $G_{s}$ is given by equation (2). With elastic mismatch,

$$
G_{\mathrm{ss}}=c_{1}^{2} \frac{\bar{\sigma}^{2} R}{E_{\mathrm{m}}}
$$

where the algorithm for computing the coefficient $c_{1}\left(f, E_{\mathrm{f}} / E_{\mathrm{m}}, v_{\mathrm{f}}, v_{\mathrm{m}}\right)$ is given by Hutchinson and Jensen [4]. On any debonded segment, the average stress in the fiber is $\bar{\sigma}_{\mathrm{f}}=\bar{\sigma} / f$. The asymptotic limit in Section 2 is expected to apply when $\Delta L$ becomes sufficiently small compared to $R$. With $\bar{\sigma}_{\mathrm{f}}$ identified with $-\sigma$, as well as $E_{1}$ and $v_{1}$ with the properties of the fiber, and $E_{2}$ and $v_{2}$ with those of the matrix, from equation (8)

$$
G=\frac{(1-\alpha) \bar{\sigma}^{2} \pi \Delta L}{16 f^{2} \vec{E}_{\mathrm{f}}},
$$

where $\bar{E}_{\mathrm{f}}-E_{\mathrm{f}} /\left(1-v_{\mathrm{f}}^{2}\right)$. Thus, as the debonds converge, with $\Delta L / R$ approaching zero,

$$
\frac{G}{G_{\mathrm{ss}}}=\frac{(1-\alpha) \pi E_{\mathrm{m}}}{16 f^{2} c_{1}^{2} \bar{E}_{\mathrm{f}}} \frac{\Delta L}{R} .
$$

When there is no elastic mismatch this reduces to

$$
\frac{G}{G_{\mathrm{ss}}}=\frac{\pi}{4(1-f)} \frac{\Delta L}{R} \text {. }
$$

A numerical evaluation of the coefficient multiplying $\Delta L / R$ in equation (17) shows that it depends weakly on the elastic mismatch, and thus equation (18) is a reasonably good approximation except for large mismatch. Specifically, for $f=0.4$, the coefficient in equation (17) is about $7 \%$ above that in equation (18) when the fiber is twice as stiff as the matrix and 7\% below when the matrix is twice as stiff as the fiber. Even when the corresponding stiffness ratios differ by a factor of five, the error in using equation (18) is only about $15 \%$.

Numerical results for $G$ normalized by $G_{s s}$ in equation (15), computed by a finite element analysis, are presented in Fig. 11 as a function of the normalized half-spacing between the converging debond crack tips, $\Delta L / R$. The calculations have been 


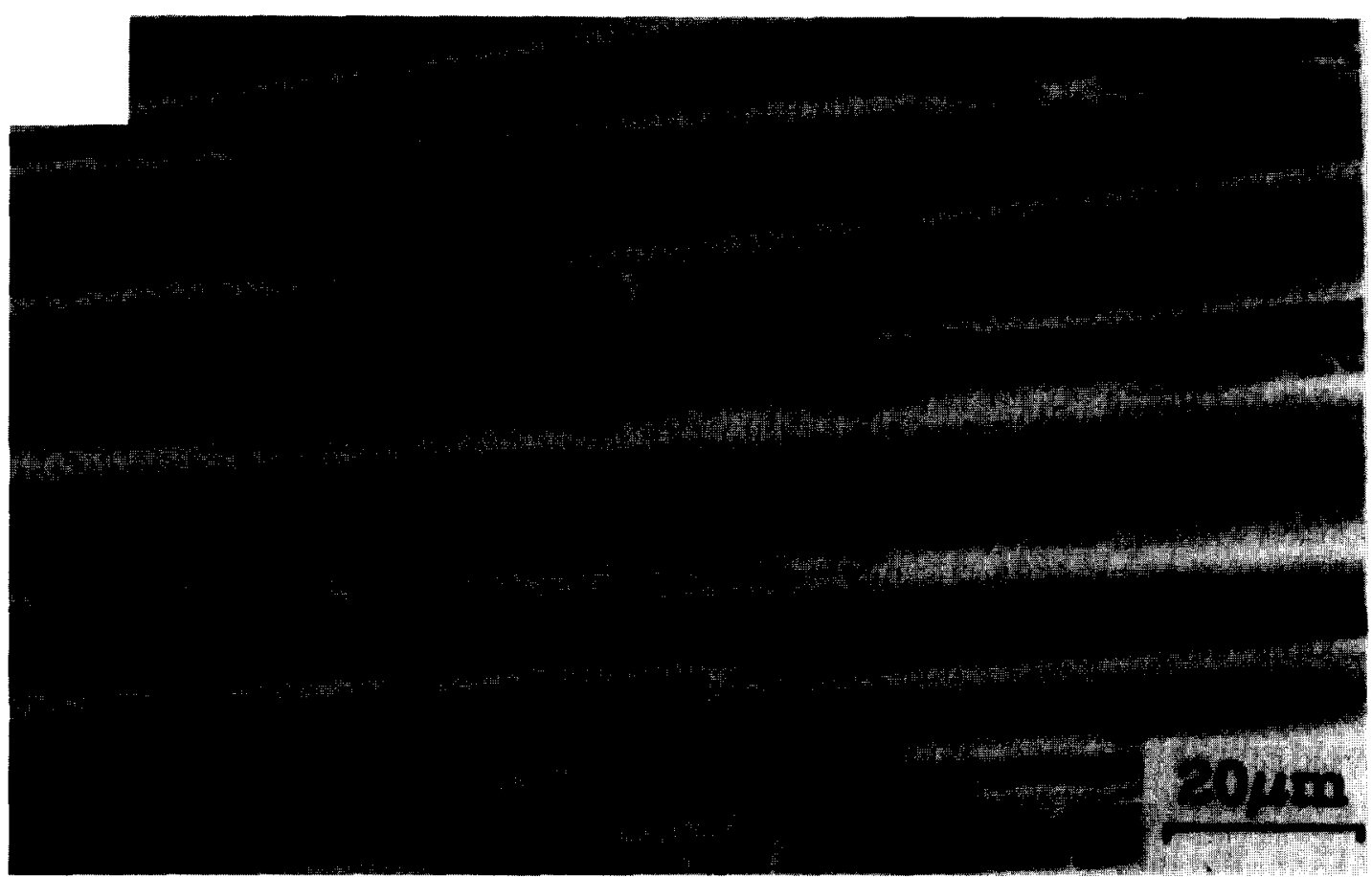

Fig. 12. A CMC transversely separated after tensile loading in the $0 / 90$ orientation up to $90 \%$ of the ultimate tensile strength. The protruberences represent the regions of intact interface as debond convergence that were ruptured upon transverse separation [13].

carried out with $f=0.4, \quad v_{\mathrm{f}}=v_{\mathrm{m}}=0.2$, and $E_{\mathrm{f}}$ ' $E_{\mathrm{m}}=1 / 2,1$ and 2 . The numerical results validate the observation that $G / G_{s s}$ has little dependence on elastic mismatch. The variation of the coefficient of $\Delta L / R$ in equation (17) over the three values of $E_{\mathrm{f}} / E_{\mathrm{m}}$ is negligible and not even observable for the asymptotic limit shown as the dashed line in Fig. 11. The numerical results show that the asymptotic limit (18) is an excellent approximation when $\Delta L / R \leqslant 1 / 2$, and that steady state persists

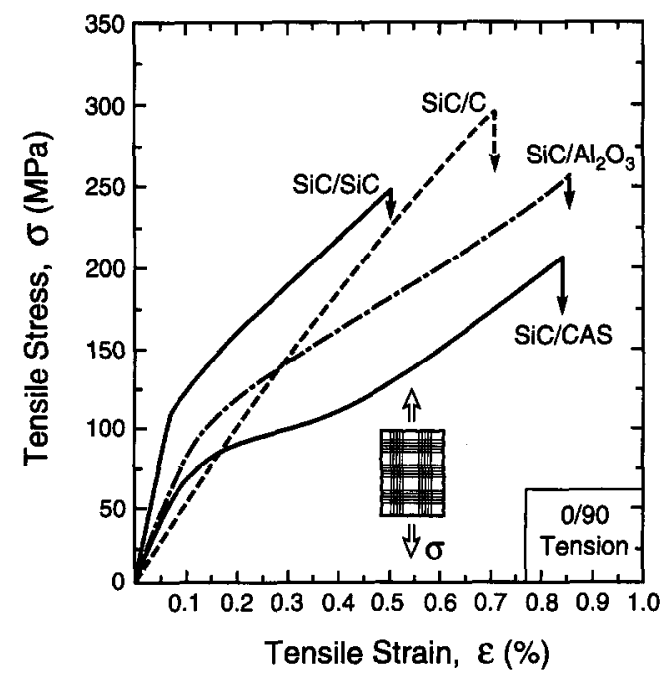

Fig. 13. Tensile stress-strain curves showing various levels of strain hardening that reflects the role of debond convergence [14] until the debond tips are within about $2 R$. Thus, the converging debonds on a fiber do not begin to sense each other until they are much closer than those for thin films.

There are two practical consequences of debond convergence. The first is that the debonds never intersect. Intact segments always exist midway between matrix cracks. This intact material becomes evident when a CMC is dissected parallel to the fibers, subsequent to tensile tests (Fig. 12). A periodic array of ridges is apparent on the dissected surface [13]. These ridges have previously been used to highlight matrix cracks and to measure crack densities. The second effect of convergence is on the effective strain hardening. As the debonds approach, the rate of inelastic straining decreases, resulting in an increase in the strain hardening coefficient. This behaviour is manifest in CMCs with low friction stress (SiC-CAS) as an upturn in the stress (Fig. 13) at larger strain levels. In CMCs with high friction (SiC-SiC), it results in a high strain-hardening rate throughout the inelastic deformation process (Fig. 13), concomitant with a larger matrix crack density [14]. It also rationalizes discrepancies found between measurements of constituent properties from hysteresis strains and simulations that neglect the convergence effect.

Acknowledgements - This work has been supported in part by the DARPA University Research Initiative through grant N00014-92-J-1808, by the NSF through grant DMR-94-00396 and by the Division of Engineering and Applied Sciences at Harvard University. 


\section{REFERENCES}

1. Evans, A. G. and Dalgleish, B. J., Acta metall mater., 1992, 40, Suppl. S295.

2. Bagchi, A., Lucas, G. E., Suo, Z. and Evans, A. G., $J$. Mater. Res., 1994, 9, 1734.

3. Zhuk, A., Evans, A. G., Whitesides, G. and Hutchinson, J. W., to be published.

4. Hutchinson, J. W. and Jensen, H. M., Mech. Mater., $1990,9,139$

5. He, M. Y., Wu, B.-X., Evans, A. G. and Hutchinson, J. W., Mech. Mater., 1994, 18, 213.

6. Rice, J. R. and Sih. G. C., J. appl. Mech., 1965, 32, 418.

7. Erdogan, F., J. appl. Mech., 1965, 32, 403.

8. Rice, J. R., J. appl. Mech., 1988, 55, 98.

9. Suo, Z. and Hutchinson, J. W., Int. J. Fracture, 1990, 43, 1

10. Drory, M. D., Thouless, M. D. and Evans, A. G., Acta metall., 1988, 36, 2019.

11. Thouless, M. D., Acta metall., 1988, 36, 3131.

12. Gao, H., Int. J. Fracture, 1990, 45, 131.

13. Cao, H. C., Bischoff, E., Sbaizero, O., Rühle, M. and Evans, A. G., J. Amer. Ceram. Soc., 1990, 73, 1691.

14. Domergue, J.-M., Heredia, F. E. and Evans, A. G., $J$. Amer. Ceram. Soc., 1996, 79, 161.

\section{APPENDIX: APPROXIMATE ANALYSIS OF THE TWO-LAYER SYSTEM}

The two-layer system with the numbering and notation for the thicknesses and moduli shown in the insert of Fig. 8 is adopted. We begin by considering the symmetric geometry shown farthest to the right in Fig. 4(a). Denote the total width of the remaining ligament by $2 b$ and the length of each crack by $a$. The pre-stress in layer 3 is $\sigma$. Analysis of the problem on the right in Fig. 4(a) will provide the energy release rate, as previously discussed, with $\sigma$ acting in the sense shown $(\sigma>0)$. Layer 1 has no pre-stress. Let $\bar{E}_{3}=E_{3} /\left(1-v_{3}^{2}\right)$ be the plane strain tensile modulus of layer 3 , while $\mu_{1}-E_{1} /\left(2\left(1+v_{1}\right)\right)$ is the shear modulus of layer 1 . If the shear stiffness of layer 1 is small compared to the tensile stiffness of layer 3 , the length of the shear lag zone through which the film stress is transferred to the substrate will be large compared to the total film thickness. We capitalize on this feature and employ a one-dimensional analysis as follows.
Let $\bar{\sigma}(x)$ be the average stress acting parallel to the film in layer 3 , with the origin of $x$ taken as the center of the remaining ligament. Direct attention to the remaining ligament, $-b<x<b$. Let $\tau(x)$ be the shear stress at the interface between layers 1 and 3 , and let $u(x)$ be the displacement of this interface in the $x$-direction. With $0^{\prime} \equiv \mathrm{d} O / \mathrm{d} x, y=u / t_{1}$ is the average shear strain in layer 1 and $\epsilon=u^{\prime}$ is the extensional strain at the bottom of layer 3. Equilibrium of layer 3 requires $\bar{\sigma}=\tau / t_{3}$. Using $\tau=\mu_{1}$ \% and $\bar{\sigma}=\bar{E}_{3} \epsilon$ in this equilibrium equation, one obtains $u^{\prime \prime}-l^{2} u=0$ where $l$ is defined in equation (14). The solution to this equation, subject to symmetry about $x=0$ and $\bar{\sigma}=-\sigma$ at $x=-b$ (or $x=+b$ ), is

$$
u=-\frac{\sigma l}{\tilde{E}_{3}} \frac{\sinh (x / l)}{\cosh (b / l)}
$$

Let $U$ be the displacement in the $x$-direction at the left end of the film at $x=-(a+b)$, and denote the potential energy of the left half of the system by PE. (Because the two halves are equal, attention is directed only to the left half.) For prescribed $\sigma$, one can readily show that $P E=-\frac{1}{2} \sigma U t_{3}$. Approximate $U$ as the sum of $u(-b)$ from equation (A.1) and $\sigma a / \vec{E}_{3}$, representing contraction of the unattached segment lying to the left of $x=-b$ :

$$
U=\frac{\sigma}{\bar{E}_{3}}\left[a+l \tanh \left(\frac{b}{l}\right)\right] .
$$

The effect of layer 1 on the contraction of the unattached segment is neglected, consistent with the assumption that layer 3 is much stiffer than layer 1 . Then, from

$$
G=-\frac{\mathrm{d} P E}{\mathrm{~d} a}=\frac{1}{2} \sigma t_{3} \frac{\mathrm{d} U}{\mathrm{~d} a} .
$$

one obtains

$$
\frac{G}{G_{\mathrm{s}}}=\tan \mathrm{h}^{2}\left(\frac{b}{l}\right)
$$

where $G_{\mathrm{ss}}=\sigma^{2} t_{3} /\left(2 \bar{E}_{3}\right)$. The final step in arriving at equation (14) is to identify $\Delta L$ (defined in the insert in Fig. 8) with $2 b$, in accordance with the discussion in the body of the paper to the effect that the tip can hardly distinguish a free edge from another debond crack. 